\title{
Primary Cutaneous Mucinous Carcinoma Treated with Narrow Surgical Margin
}

Jin-Hee Choi,

Seung Chan Kim,

Jiye Kim,

Yoon Kyu Chung

Department of Plastic and Reconstructive Surgery, Yonsei University Wonju College of

Medicine, Wonju, Korea

No potential conflict of interest relevant to this article was reported.
Primary cutaneous mucinous carcinoma (PCMC) is a rare malignant tumor of eccrine origin. Clinically, the carcinoma presents as a solitary, slow growing, and painless nodule. For this reason, this tumor is often considered to be a benign mass in the preoperative setting. The lesion is, however, malignant in nature and has a tendency for local recurrence and infrequent metastasis. Wide local excision is the treatment of choice. However, few reports exist with information regarding surgical margins and clinical outcomes. Herein, we report a case of PCMC excised with a narrow surgical margin and review the relevant literature. A 49-year-old man presented with a small cutaneous nodule of the right cheek. The mass was excised without any margin, but pathologic examination revealed histology of mucinous carcinoma. Because of this, the operative site was re-excised with a 5-mm margin, and the wound was closed using a V-Y advancement flap. Systemic work-up did not reveal other potential metastatic primary, for a final diagnosis of $\mathrm{PCMC}$. We report this case of PCMC, treated with relatively narrow margin in a patient with good prognostic factors.

Keywords: Adenocarcinoma / Mucinous adenocarcinoma / Primary mucinous carcinoma

\section{INTRODUCTION}

Primary cutaneous mucinous carcinoma (PCMC) is a rare malignant tumor of eccrine origin with only about 150 cases reported [1,2]. Clinically, the carcinoma presents as a solitary, slow growing, and painless nodule [2,3]. For this reason, the lesion is often regarded as a benign mass at the initial presentation. In a vast majority of cases, a two-stage operation was needed for pathologic confirmation of clear surgical margins because of inadequate resection margin at the initial operation $[3,4]$.

Histologic examination is not able to distinguish between primary and metastatic mucinous carcinoma of the skin. Most mucinous carcinomas in the skin are metastatic lesions, and histologic diagnosis of mucinous carcinoma always warrants a

\section{Correspondence: Yoon Kyu Chung}

Department of Plastic and Reconstructive Surgery, Wonju Severance Christian Hospital, Yonsei University Wonju College of Medicine, 20 Ilsan-ro, Wonju 26426, Korea E-mail: ykchung@yonsei.ac.kr

Received May 17, 2016 / Revised August 16, 2016 / Accepted August 16, 2016 systemic workup for a potential primary source, especially of the breast or the gastrointestinal tract. Few reports exist with information regarding surgical margins and clinical outcomes. Herein, we report a case of PCMC excised with a narrow surgical margin and review the relevant literature.

\section{CASE REPORT}

A 49-year-old Korean male patient presented with a palpable nodule of the right cheek (Fig. 1). The nodule was a firm, nontender, subcutaneous mass. His medical history was significant for a motorcycle collision 13 years ago, from which he suffered a right-sided facial injury and loss of vision in the right eye. More recent than this, the patient suffered a bicycle collision 2 years prior to presentation and underwent open reduction of a right zygomaticomaxillary fracture. One year after the bicycle accident, the patient began to notice a small nodule in the traumatized area and slowly grew. 
Initially, we believed the lesion to be an epidermal cyst or another mass of benign origin. The mass was excised under local anesthesia without much consideration to tumor margins. Intraoperatively, the tumor was situated just underneath the dermis with capsulation. The mass was dissected from the skin, and no skin excision was necessary to isolate and resect the mass. Grossly, the $6 \times 5 \times 4 \mathrm{~mm}$ tumor was yellow-brown in color with a smooth encapsulating surface (Fig. 2). Histopathologic examination revealed the tissue to contain mucinous lakes separated by fibrous septa (Fig. 3). Neoplastic cells were round to cuboidal with abundant cytoplasm and minimal atypia. Cribriforming and small glandular structures were also noted. On immunohistochemistry, the neoplastic cells were strongly positive for cytokerain 7 (CK-7), gross cystic disease fluid protein 15, and mucin 1 protein. The cells were negative for cytokeratin 20 (CK-20) and mucin 5AC. These morphologic and immunohistochemical features were consistent with mucinous adenocarcinoma.

Because of the unexpected finding of mucinous carcinoma, an

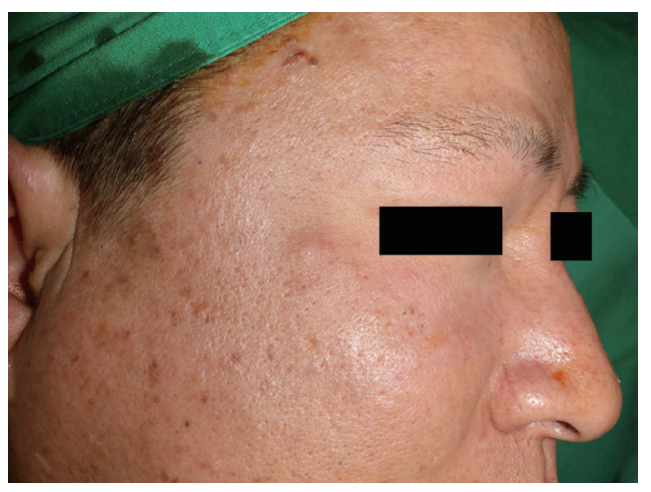

Fig. 1. A 49-year-old male presented with a palpable nodule of the right cheek.

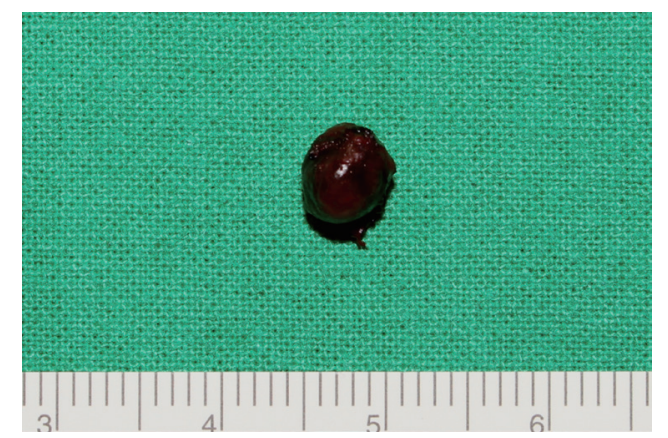

Fig. 2. Gross morphology of primary cutaneous mucinous carcinoma. extensive oncologic evaluation was performed to evaluate for any extracutaneous metastatic primary (i.e., breast or gastrointestinal tract). The evaluation included computed tomography of the head and neck, a positron emission tomographic scan, and an upper and lower gastrointestinal tract endoscopy. No extracutaneous source was found, and the diagnosis of a PCMC was confirmed.

At 3 weeks after the first operation, the patient underwent a wide local excision with a safety margin of $5 \mathrm{~mm}$, and was found to have clear surgical margin on frozen section. The resulting $1 \times$ $1.5 \mathrm{~cm}$ defect was covered with a croissant -shaped modified V-Y advancement flap (Fig. 4). Considering the lack of any evidencebased data regarding the treatment and follow-up of this rare tumor, the patient was followed every 6 months for early detection of local recurrence or metastasis. The follow-up workup included

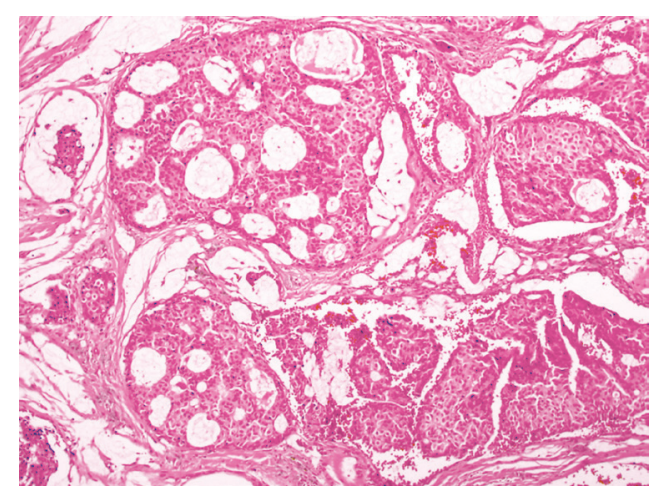

Fig. 3. Histologic examination of the first surgical specimen reveals clusters of tumor cells and mucinous lakes separated by fibrous septa $(\mathrm{H} \& \mathrm{E}, \times 100)$.

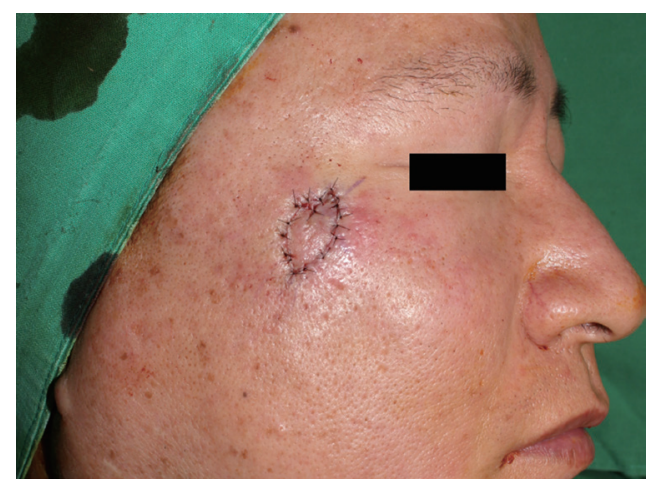

Fig. 4. The defect was covered with a croissant-shaped modified V-Y advancement flap after wide excision. 


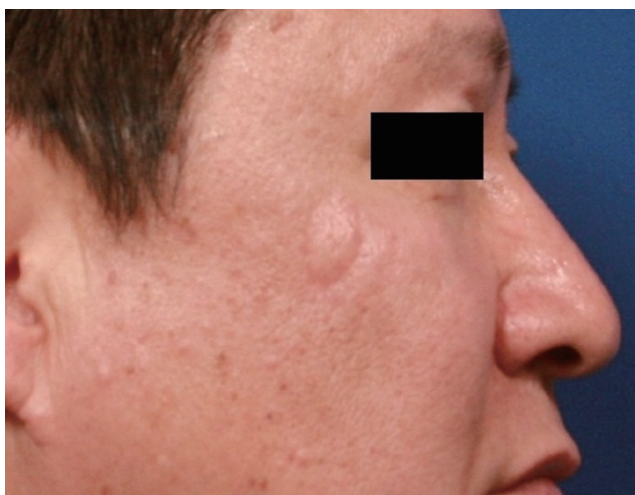

Fig. 5. After one year, no evidence of recurrence was present on physical examination.

physical examination, computed tomography (CT) scan, and CA $72-4$, which is a tumor marker for detecting tumor-associated glycoprotein 72 (TAG-72), a mucin-like molecule found on the surface of cancer cells [5]. The patient had no evidence of recurrence or metastasis during a 14-month follow-up (Fig. 5).

\section{DISCUSSION}

PCMC, an uncommon malignancy, was first described by Lennox et al. [1] in 1952. There are no characteristic clinical features to allow easy recognition of this tumor. Therefore, differential diagnosis of PCMC from any other neoplasm is difficult before pathologic confirmation, but its histopathologic findings are unique and show similar patterns in most cases [4]. Still, differential diagnosis from metastatic mucinous adenocarcinoma is essential for diagnostic confirmation of PCMC. Exclusion of primary carcinoma in the breast, gastrointestinal tract, or other organs needs to be specifically sought after. Immunohistochemistry based on expression of cytokeratins can be helpful to rule out any other metastases. PCMC is CK-7 positive and CK-20 negative, unlike metastases of gastrointestinal tract which are CK-20 positive and CK-7 negative [4]. Although helpful in our case, these diagnostic markers cannot exclude metastatic disease with $100 \%$ certainty. Other diagnostic work-up such positron emission tomography/CT and upper and lower gastrointestinal endoscopy are needed.

PCMC is considered a low-grade malignant tumor because metastasis to other organ or regional lymph nodes is very rare. However, inadequate safety margin is associated with recurrence rates between $20 \%$ and $30 \%$. As such, the gold standard of treatment is local wide excision [2]. In the case presented, the $0.5-\mathrm{cm}$ surgical margin is smaller than in previous reports, in which the skin tumors were resected with the traditional $1-\mathrm{cm}$ surgical margin (ranged from 0.2 to $2.0 \mathrm{~cm}$ ). We had few reasons for this smaller margin. First, the primary lesion was a very small nodule $(0.6 \times 0.5 \times 0.4 \mathrm{~cm})$, which was a good prognostic factor [2]. Second, pathologic examination estimated the first specimen to contain clear margin, and intra-operative frozen section was clear even with the small margin. Finally, the age and ethnicity of the patient also portended a favorable prognosis. According to a recent metaanalysis by Kamalpour et al. [2], lower rates of metastasis and recurrence have been reported among younger patients and East Asians. They also reported that head and neck (compared to the trunk) lesions was predictive of a good outcome, which is in contrast to other non-melanomatous skin cancers like squamous cell and basal cell carcinomas. Moreover, the tumor size influenced the poor outcome in their meta-analysis.

Despite the lack of a long-term follow up, our patient is the first case report in which the margin was selected based on prognostic factors. We also presented the specific follow-up workup, which had not been reported in any other study. We propose that, for patients with good prognostic factors, the tissue-sparing surgery with narrow margin can be appropriate, especially for a facial lesion.

\section{REFERENCES}

1. Lennox B, Pearse AG, Richards HG. Mucin-secreting tumours of the skin with special reference to the so-called mixed-salivary tumour of the skin and its relation to hidradenoma. J Pathol Bacteriol 1952;64:865-80.

2. Kamalpour L, Brindise RT, Nodzenski M, Bach DQ, Veledar E, Alam M. Primary cutaneous mucinous carcinoma: a systematic review and meta-analysis of outcomes after surgery. JAMA Dermatol 2014;150:380-4.

3. Requena L, Mengeesha Y, Kutzner H, et al. Appendageal tumors. In: LeBoit PE; International Agency for Research on Cancer; World Health Organization, et al., editors. Pathology and genetics of skin tu- 
mours. Lyon: IARC Press; 2006. p. 132-3.

4. Adefusika JA, Pimentel JD, Chavan RN, Brewer JD. Primary mucinous carcinoma of the skin: the Mayo Clinic experience over the past 2 decades. Dermatol Surg 2015;41:201-8.
5. Guadagni F, Roselli M, Cosimelli M, Spila A, Cavaliere F, Tedesco M, et al. Correlation between tumor-associated glycoprotein 72 mucin levels in tumor and serum of colorectal patients as measured by the quantitative CA 72-4 immunoassay. Cancer Res 1996;56:5293-8. 